CERIAS Tech Report 2001-28

\title{
1THz Repetition Rate Multichannel Pulse Train Source using an Arrayed Waveguide Grating
}

D.E. Leaird ${ }^{1}$, S. Shen ${ }^{1}$, A.M. Weiner ${ }^{1}$, A. Sugita ${ }^{2}$ H. Yamada ${ }^{2}$, S. Kamei ${ }^{2}$, M. Ishii ${ }^{2}$, K. Okamoto ${ }^{2}$ Center for Education and Research in Information Assurance and Security $\&$

${ }^{1}$ School of Electrical \& Computer Engineering, Purdue University West Lafayette, IN 47907

${ }^{2}$ NTT Photonics Laboratories 


\title{
1 THz Repetition Rate Multichannel Pulse Train Source using an Arrayed Waveguide Grating
}

\author{
D.E. Leaird ${ }^{1,2}$, S. Shen ${ }^{1}$, A.M. Weiner ${ }^{1,2}$ \\ ${ }^{I}$ School of Electrical \& Computer Engineering, Purdue University, West Lafayette, IN 47907-1285, USA \\ ph:765-494-3370,fax: 765-494-6951,leaird@purdue.edu, shuai@ecn.purdue.edu, amw@ecn.purdue.edu \\ ${ }^{2}$ Center for Education and Research in Information Assurance and Security - CERIAS \\ Purdue University, West Lafayette, IN 47907 \\ A. Sugita, H. Yamada, S. Kamei, M. Ishii, and K. Okamoto \\ NTT Photonics Laboratories, 162 Tokai, Naka-gun, Ibaraki-pref., 319-1193, Japan \\ sugita@iba.iecl.ntt.co.jp,kamei@iba.iecl.ntt.co.jp, ishii@iba.iecl.ntt.co.jp, okamoto@iba.iecl.ntt.co.jp
}

\begin{abstract}
THz}$ repetition rate bursts of short pulses at multiple spatially separated output channels are demonstrated from a single short pulse source laser and an arrayed waveguide grating designed to have a free spectral range less than the bandwidth of the source laser.

(C)2000 Optical Society of America

OCIS codes: (060.2380) Fiber optics sources and detectors; (320.7160) Ultrafast technology;

(130.3120) Integrated optics devices
\end{abstract}

The Arrayed-Waveguide Grating (AWG) has seen considerable development in the past few years 112 primarily in the development of devices for use as Wavelength Division Multiplexed (WDM) channel demultiplexers and routers. In contrast AWG devices have seen only limited use in time domain applications. For example, modelocked pulse inputs have been spectrally sliced to yield pulses in the tens of picoseconds range at the repetition rate of the modelocked source laser [3], and supercontinuum sources have been sliced to yield multiple optical wavelengths for high-speed systems studies [4,5]. Modified AWG devices have also been used for Fourier transform optical pulse shaping [6]. Here we present a completely new functionality, in which the AWG produces bursts of pulses repeating at a $\mathrm{THz}$ rate, with the pulse repetition rate determined by the AWG design rather than the input pulse repetition rate. Furthermore, wavelength shifted but otherwise identical pulse bursts are obtained at different output channels of the AWG, very much like the direct space-to-time (DST) pulse shaper [79], previously investigated only in bulk optics. Continuous trains of very high repetition rate pulses from a lower repetition rate source should be possible with proper selection of the source laser and AWG design.

For the generation of very high repetition rate pulse bursts, the important AWG parameter determining the character of the output is the free spectral range (FSR), which is equal to the inverse of the delay increment per guide $(\Delta \tau)$ in the waveguide array:

$$
\mathrm{FSR}=\Delta \tau^{-1}=\frac{\mathrm{c}}{\mathrm{n}_{\text {eff }} \Delta \mathrm{L}}
$$

where $\mathrm{n}_{\text {eff }}$ is the effective index of the waveguide, $\mathrm{c}$ is the speed of light in vacuum, and $\Delta \mathrm{L}$ is the physical path length difference from one guide to the next in the waveguide array section of the AWG. In traditional AWG devices, the FSR is typically required to be large to ensure that a unique output wavelength within the DWDM system is present at each AWG output. For the generation of THZ rate trains of pulses, we work in the opposite regime, where the optical bandwidth of the source laser, $\Delta \mathrm{v}$, exceeds the FSR. If the input pulses are bandwidth limited, this means the input pulse width, $\mathrm{t}_{\mathrm{p}}$, is less than the delay increment per guide, $\mathrm{t}_{\mathrm{p}}<\Delta \tau$.

In this limit, the output spectrum on a single output guide is multiply peaked, with the spectral peak spacing equal to the FSR. Accordingly, in the time domain, this corresponds to a pulse train with a pulse separation, $\Delta \mathrm{t}$, equal to the waveguide delay increment, $\Delta \mathrm{t}=\mathrm{FSR}^{-1}=\Delta \tau$. Thus, the waveguide array acts as a series of delay lines, and each pulse in the output train can be identified with propagation through a specific guide in the array. This realization may permit tailored output temporal profiles by engineering the waveguide array loss on a guide-by-guide basis as will be discussed later. The duration of the individual output pulses is the same as the input, while the duration of the envelope of the burst of pulses, $\mathrm{T}$, varies inversely with the AWG passband width, $\delta \mathrm{f}$. 
In the experiments described here, a modelocked erbium fiber laser producing a $50 \mathrm{MHz}$ train of $\sim 200$ fs pulses at $1560 \mathrm{~nm}$ is utilized as the source laser. In principle, similar experiments can also be performed using much higher rate (e.g. $>10 \mathrm{GHz}$ ) modelocked pulse sources, which may result in continuous or quasi-continuous output pulse trains. The laser output is split with a 10/90 fiber splitter, and all fiber links are constructed to be dispersion compensated using an appropriate combination of single mode fiber and dispersion compensating fiber. The $90 \%$ arm is launched into an Arrayed Waveguide Grating (AWG) that has been designed to have a relatively small free spectral range (FSR) of $1 \mathrm{THz}$ (corresponding to a relatively large delay increment of $\Delta \tau=1 \mathrm{ps}$ ). The temporal profile of the AWG outputs are recorded via intensity cross correlation in a free-space apparatus using the $10 \%$ port of the fiber splitter as a reference pulse. Power spectra of the AWG outputs are recorded as well with an optical spectrum analyzer. Two different AWG's were investigated. One device has $100 \mathrm{GHz}$ channel spacing and 10 output channels, while the second has $40 \mathrm{GHz}$ channel spacing and 25 output channels.

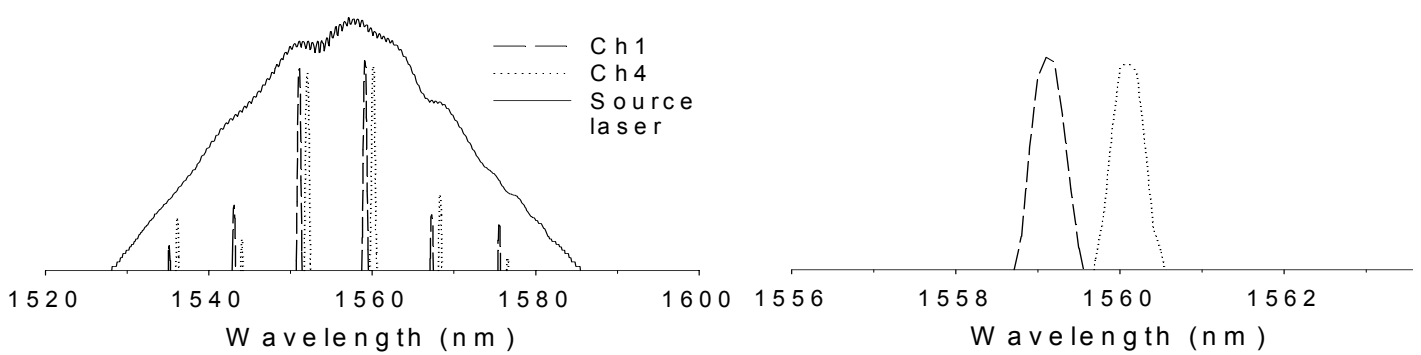

Fig. 1 Power spectra from the $40 \mathrm{GHz}$ output channel spacing AWG for channel 1 (dash), and channel 4 (dot).

The source laser is indicated by the solid line. Both curves are plotted on a semi-log axis.

Fig. 1 shows power spectra from the $40 \mathrm{GHz}$ output channel spacing device. In Fig. 1A, the power spectrum of the source laser is overlaid with the output power spectra recorded from channels 1 and 4 . The spectra consist of six discrete output frequencies within the bandwidth of the source laser for each output channel. The $1 \mathrm{THz}$ FSR is evident from the periodic passband structure with $8.1 \mathrm{~nm}$ spacing between peaks. The shift in output center wavelength from one output to another is apparent as well. In order to more clearly show the shift in output center wavelength across the output guides, Fig. 1B shows an expanded version of the central passbands. The $\sim 0.95 \mathrm{~nm}$ spacing between the two output channels corresponds to an average $39.1 \mathrm{GHz}$ channel spacing per output. The multiply peaked nature of the power spectra demonstrate that $\Delta v>$ FSR; therefore, the output temporal profile is expected to be a train of pulses where the temporal period of the pulses in the output train is given by the inverse of the FSR.

Fig. 2 shows intensity cross correlation measurements of two different AWG devices - the $40 \mathrm{GHz}$ output channel spacing device (right side of Fig. 2) used in the power spectra measurements of Fig. 1, and a $100 \mathrm{GHz}$ output channel spacing device (left side of Fig. 2) for two different output channels of each device. As expected from the power spectrum presented above the temporal profile consists of a train of
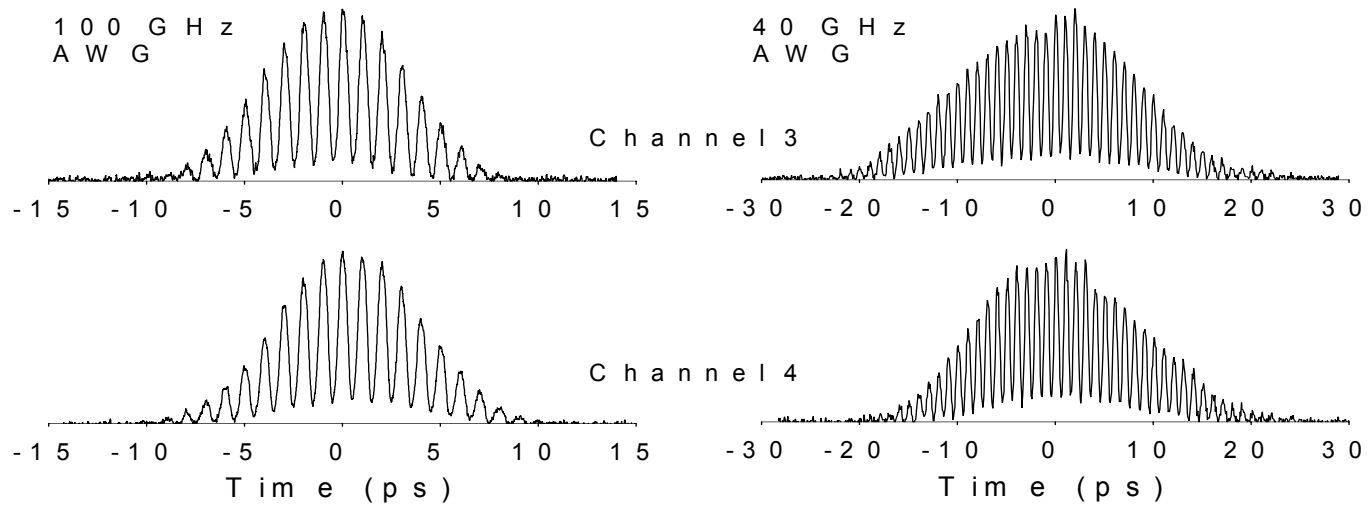

Fig. 2 Intensity cross correlation measurments from the $100 \mathrm{GHz}$ output channel spacing (left), and $40 \mathrm{GHz}$ output channel spacing (right) AWG's for output channel 3 (top) and 4 (bottom). 
pulses with a $1 \mathrm{ps}$ period ( $1 \mathrm{THz}$ repetition rate), corresponding to the AWG delay increment, which is the inverse of the FSR, and with a pulse duration comparable to that of the input pulse. The temporal window (or number of pulses) is inversely proportional to the AWG filter bandwidth and therefore increases by 2.5 times from the $100 \mathrm{GHz}$ device to the $40 \mathrm{GHz}$ device. The form of the output intensity profile is the same for different output channels of a single device, even though the spectrum is shifted.

We have performed initial work on further tailoring the output temporal profile of such pulse bursts, with the goal of generating a flat-topped train of output pulses, as opposed to the pulse train under a Gaussian envelope as demonstrated above. It should be possible to achieve this goal by tailoring the loss within the waveguide array on a guide-by-guide basis. Fig. 3 shows measurements of the electric field amplitude [10] of light emerging from different guides within a single custom waveguide array fabricated for this purpose. The losses have been adjusted to give close to equal amplitudes over 21 different guides, which should result in generation of nearly flat-topped pulse trains. We plan to pursue further time-domain experiments testing this expectation.

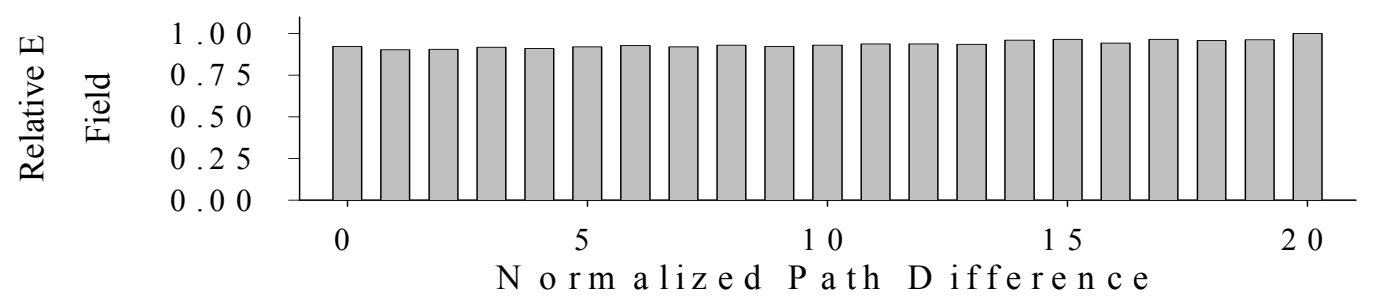

Fig. 3 Measured electric field amplitude in an AWG device designed to have a flat-topped output temporal intensity profile.

In summary, $1 \mathrm{THz}$ repetition rate pulse bursts have been demonstrated from a specially designed AWG and a single short pulse source laser. The key requirement is that the FSR of the device must be tailored so that multiple filter passbands fit within the input laser bandwidth. The unique properties of this device allow generation of identical, wavelength shifted, very high rate pulse trains for hybrid TDM/WDM communications and photonic signal processing. Initial work has been performed on tailoring the output temporal profile to generate a flat-topped pulse train burst. When applied to a high-repetition rate modelocked source $(>10 \mathrm{GHz})$, these devices could lead to continuous very-high repetition rate output trains $(>500 \mathrm{GHz})$.

This material is based upon work supported by, or in part by the U.S. Army Research Office under contract DAAG55-98-1-0514, and by sponsors of the Center for Education and Research in Information Assurance and Security. Also, we thank IMRA America for the loan of the femtosecond modelocked fiber laser.

\section{References}

1. R. Adar, C.H. Henry, C. Dragone, R.C. Kistler, M.A. Milbrodt, "Broad-band array multiplexers made with silica wave-guides on silicon," J. Lightwave Tech., 11, 212-219, (1993).

2. K. Okamoto, "Recent progress of integrated optics planar lightwave circuits," Opt. and Quant. Elec. 31, 107-129 (1999).

3. I.Y. Khrushchev, J.D. Bainbridge, J.E.A. Whiteaway, I.H. White, and R.V. Petty, "Multiwavelength pulse source for OTDM/WDM applications based on arrayed waveguide grating," IEEE Photon. Tech. Lett., 11, 1659-1661 (1999).

4. T. Morioka, K. Uchiyama, S. Kawanishi, S. Suzuki, and M. Saruwatari, "Multiwavelength picosecond pulse source with low jitter and high optical frequency stability based on 200nm supercontinium filtering," Electron. Lett., 31, 1064-1066 (1995).

5. S. Kawanishi, H. Takara, K. Uchiyama, I. Shake, and K. Mori, "3 Tbit/s (106 Gbit/s x 19 channel) optical TDM and WDM transmission experiment," Electron. Lett. 35, 826-827 (1999).

6. T. Kurokawa, H. Tsuda, K. Okamoto, K. Naganuma, H. Takenouchi, Y. Inoue, and M. Ishii, "Time-space-conversion optical signal processing using arrayed-waveguide grating,” Electron. Lett. 33, 1890-1891 (1997).

7. B. Colombeau, M. Vampouille, and C. Froehly, "Shaping of Short Laser Pulses by Passive Optical Fourier Techniques," Opt. Comm., 19, 201-204 (1976).

8. D.E. Leaird, and A.M. Weiner, "Femtosecond optical packet generation by a direct space-to-time pulse shaper," Opt. Lett. 24, 853-855 (1999).

9. D.E. Leaird, and A.M. Weiner, "Wavelength tunable femtosecond pulse trains from a direct space-to-time pulse shaper," in Conference on Lasers and Electro-Optics, OSA Technical Digest, 410-411 (2000).

10. K.Takada, Y.Inoue, H.Yamada and M.Horiguchi, "Measurement of phase error distributions in silica-based arrayed-waveguide grating multiplexers by using Fourier transform spectroscopy," Electron. Lett., 30, 1671-1672 (1994). 